\title{
La littérature à l'épreuve du réel : les jeux de Pascal Quignard avec les Lettres
}

\author{
Xavier Martin (Université d'Oulu, Finlande)
}

\begin{abstract}
The novel Les Larmes, by French writer Pascal Quignard, takes place in the ninth century, two twin brothers make totally different choices of life. Hartnid is always on the go. His twin, Nithard, is a pure scholar. His life is devoted to language. It is precisely his involment in language that allows him to write something that was never written before. He writes the Serments de Strasbourg (English: Oaths of Strasbourg, Latin: Sacramenta Argentariae) in 842 , first written marks of a language that will become the French language. This novel offers a unique opportunity to sketch a thought on the ability of literature to speak about our relationship to reality.

The first part of the article questions the gesture of the novelist who acts on himself and on reality when writing, he really tries to change the world.

The second part of the article studies the notion of realism and shows how Quignard finds a place in the history of literature that, today, stays away from realism, but, maybe, in order to better track down reality. Finally, in order to grasp the specificity of the Works of Quignard, my interest in the aesthetic of Les Larmes is threefold: its complex structure, the fascination for origin that it shows and, the question of identity and splitting with the twin brothers Hartnid and Nithard.

The article concludes on the ability of artists to anticipate in their creations and, in advance, to tell about events that will occur in the future.
\end{abstract}

Keywords: French literature, Pascal Quignard, French language, fiction, realism

\section{Introduction}

Cet article tient en partie à la formulation de la thématique de la $20^{\mathrm{e}}$ Conférence des Romanistes Nordiques, la question des migrations. Il repose aussi sur le principe même qui nous réunit : la notion de langues romanes. En effet, il se trouve que Pascal Quignard a publié un roman, Les Larmes, qui parle de peuples cherchant à conquérir de nouveaux territoires, et fait état de combats, de chocs des cultures :

Au même moment deux assauts s'associèrent et étranglèrent l'Europe comme des crocs. Une invasion progressive, savante, subtile, pieuse, au sud, une invasion brutale, barbare, cupide, violente, au nord. (Quignard 2016, 26)

Les raids terribles et imprévisibles des Normands venaient relayer les razzias des Arabes et amplifiaient la dévastation de tous bords, sur tous les fleuves, sur toutes les mers, sur tous les confins, jusque dans les montagnes. (Quignard 2016, 30) 
Ce texte parle aussi de l'émergence de la langue française, et c'est ce qui va m'intéresser plus précisément, de la naissance de l'écriture de cette langue et de la littérature qui va s'y développer.

Dans Les Larmes, Pascal Quignard met en avant deux textes : ce que l'histoire retient comme les «Serments de Strasbourg » écrits par Nithard en 842, première trace écrite de la langue française et, la «Séquence de sainte Eulalie » présentée comme la première œuvre de la littérature française, cantique écrit en 881. Quignard présente la date et presque l'heure de l'écriture de ces textes. Il se saisit de ces deux événements et, d'une certaine manière, se les approprie par l'écriture. Il ne cherche pas à faire un travail d'historien, il écrit un roman ; «roman» est le terme qui apparaît sous le titre du livre. Les Larmes est une œuvre particulièrement complexe, elle est composée de faits historiques avérés, de faits liés à l'histoire personnelle de Quignard, d'aventures vécues par des personnages fictifs ou bien d'aventures fictives vécues par des personnages réels. Bref, Quignard fait de la littérature, il fabrique un texte, à partir de ce qu'il sait de la naissance de l'écriture du français et de la littérature française ; il invente une série d'histoires à partir de là.

C'est de cette complexité de l'œuvre que je vais esquisser une réflexion sur la nature du rapport entre la littérature et le réel. Je vais procéder en trois temps. Dans un premier temps, je vais poser la question : Pourquoi écrire, pourquoi utiliser la littérature pour aborder des faits historiques? Quels liens peut-on établir entre la littérature et le réel ? Que cherche à faire l'écrivain quand il écrit? Dans cette partie, je vais m'appuyer principalement sur des écrits de Quignard, sur quelques-unes de ses déclarations mais aussi sur des propos d'autres écrivains ou critiques littéraires. Dans un deuxième temps, par souci de clarté, je vais revenir sur la notion de réalisme dans la littérature. Je vais m'appuyer principalement sur une étude Les romanciers du réel. De Balzac à Simenon, de Jacques Dubois (Dubois 2000) pour tenter de saisir l'approche de Quignard. Il ne se situe pas dans une quelconque approche réaliste, le terme «roman» associé à son texte le montre clairement. Mais revenir sur la question du réalisme permettra, je l'espère, de saisir des partis-pris forts de l'œuvre. Dans un troisième temps, je vais étudier quelques caractéristiques de l'esthétique de Quignard dans Les Larmes. Le roman a, en effet, une forme particulièrement complexe. Je vais m'arrêter sur trois aspects singuliers de son roman : 1 . La division en chapitres très hétérogènes : ils peuvent présenter des faits historiques à la manière d'un historien ou d'un érudit, ou laisser la place à un " je » qui évoque des moments de son histoire personnelle. Ils peuvent encore raconter des aventures de personnages au statut incertain. Le roman glisse même vers le fantastique à d'autres moments. -2 . La fascination pour l'origine et le moment de naissance : ici naissance du français, de son écriture, naissance de la littérature française. Quignard retrouve le moment où tout a basculé, l'instant où tout a changé, où plus rien ne sera plus jamais comme avant. 3. La question de l'identité à travers une forme de dédoublement des personnages : dans Les Larmes, Nithard et Hartnid sont des jumeaux que tout oppose. Hartnid fait le choix de vivre, de voyager, d'aimer. L'existence de Nithard semble se réduire à l'espace de la cellule où il travaille. L'écrivain, pour écrire, a besoin de se couper du monde ? Cette opposition entre l'écrivain, accaparé par le langage, y consacrant tout son temps, et le reste des vivants est fréquente dans l'œuvre de Quignard.

Dans la conclusion, pour ouvrir la réflexion, je dirai quelques mots sur la notion d'identité narrative développée par Paul Ricœur dans Temps et récit (Ricœur 1983-1985) et sur l'essai Le Titanic fera naufrage de Pierre Bayard (Bayard 2016) qui donne le cadre général d'une réflexion sur les relations entre la littérature et le réel, questionne la capacité 
des romanciers et des artistes à anticiper et décrire à l'avance des événements qui se produiront dans la réalité.

\section{Pourquoi écrire ?}

Pascal Quignard n'aborde pas directement la question de son écriture dans Les Larmes, mais il le fait dans d'autres textes. Ces autres textes doivent nous aider à lire le roman car, comme l'a suggéré Chantal Lapeyre-Desmaison dans Mémoires de l'origine. Un essai sur Pascal Quignard, on devrait «lire l'œuvre : non comme des ouvrages réellement autonomes, mais comme un ensemble, dont chaque élément n'est qu'une réalisation particulière. » (Lapeyre-Desmaison 2001, 205). En vertu de ce principe, je vais chercher dans d'autres publications de Quignard des réponses à ce questionnement sur le pourquoi de l'écriture. Quignard aborde ce thème dès les premières lignes de l'avertissement de Dans ce jardin qu'on aimait :

une dépression toute venimeuse et douce, brumeuse, insinuante, saisonnière, tourne
autour de moi comme un nuage vague, finalement me prend. Puis elle s'appesantit. Elle
se fait presque lourde, elle se transforme peu à peu en une véritable tristesse. Alors il me
faut inventer une tâche. Il me faut tromper le temps, leurrer les heures, entrer dans mon
lit, détripler les oreillers, amonceler les couvertures, repousser le froid. C'est ainsi qu'en
1989 j'ai eu le désir de raconter la vie d'un musicien qui me paraissait mal connu.
(Quignard 2017,7)

Deux éléments se dégagent de cet extrait : Quignard écrit pour lutter contre la dépression saisonnière qui le touche chaque année. Déjà dans Critique du jugement (Quignard 2015), il avait évoqué l'écriture et la publication comme des moyens de lutter contre la dépression. Et, deuxième élément, Quignard a éprouvé "le désir de raconter la vie d'un musicien [...] mal connu »; Quignard évoque ici Sainte Colombe que son roman Tous les matins du monde (Quignard 1991) et le film d'Alain Corneau (Corneau 1991) ont contribué à faire connaître auprès d'un vaste public. Dans ce jardin qu'on aimait (Quignard 2017) se situe explicitement dans le prolongement de Tous les matins du monde (Quignard 1991) puisque l'ouvrage met en scène un autre musicien, l'américain Simeon Pease Cheney (18181890), «premier compositeur moderne à avoir noté tous les chants des oiseaux qu'il avait entendus » (Quignard 2017, quatrième de couverture). Quignard précise encore :

Cette double histoire [...] prit en moi la forme non pas d'un essai ni d'un roman, mais d'une suite de scènes amples, tristes, lentes à se mouvoir, polies, tranquilles, cérémonieuses, très proches des spectacles de nô du monde japonais d'autrefois. (Quignard 2017, 10-11)

On pourrait très facilement dire aussi des Larmes que c'est une suite de scènes... De l'avertissement de Dans ce jardin qu'on aimait, il se dégage qu'écrire pour Quignard, c'est aussi faire revenir des morts - c'est un peu le principe du théâtre nô -, et leur donner une nouvelle vie. Sans aller jusqu'à faire une archéologie des textes de Quignard, on peut noter une évolution de cette thématique récurrente chez notre auteur. Il passe de la surprise dans $L e$ Nom sur le bout de la langue : "Il est curieux de noter que les livres que j'ai écrits ont connu le succès en déterrant de vieux fantômes morts inconnus qui portaient en eux plus d'avenir que des vivants. » (Quignard 1993, 61) ; à la reconnaissance d'une démarche délibérée dans La Haine de la musique : «J'ai essayé de faire revenir des choses qui étaient sans code, sans 
chant et sans langage et qui erraient vers la source du monde.» (Quignard 1996, 275) ; à l'affirmation d'une nécessité dans Sur le jadis : «Il faut sans cesse remonter à la lumière d'un nouveau jour de nouveaux morts où la vie au hasard de la mémoire s'est déplacée et se concentre. » (Quignard 2002, 297); et d'une recherche volontaire :

Depuis l'âge de dix-neuf ans, depuis le premier livre que j'ai écrit et qui portait sur Maurice Scève, j'aurai cherché à faire revenir du monde des ombres des figures dédaignées, difficiles, fascinantes, ombrageuses, butées, splendides. Scève, Lycophron, Albucius, [...] À chacun des livres que je leur consacre la conviction me porte que je vais effacer un peu l'infamie de l'Histoire, réparer l'erreur, apaiser l'errance, arracher le langage à son destin de calomnies, de bégueuleries, de paix, de chantonnement aigre, de gémissement tremblant, assourdi, chevrotant. (Quignard 2002, 296)

Ici Quignard se présente comme quelqu'un qui lutte contre l'amnésie de l'Histoire, il se bat pour sortir des auteurs de l'oubli. Son écriture a le pouvoir de ressusciter des morts injustement négligés, elle n'est donc pas simplement le fait de la dépression automnale. Quignard avance un autre argument dans Sur le jadis ; écrire, mais c'est valable pour tous les arts, provoque une joie, la joie de jouer avec le perdu, d'en faire surgir des fragrances :

De même au sein de chaque œuvre dans chaque art la fascination qui s'y cherche procure
l'émotion esthétique suivante : la joie de faire resurgir à des millénaires ou à des siècles
de distance le perdu lui-même, la sensation de retrouver avec le perdu en personne, la
nostalgie de ce qui a cessé d'être, l'épiphanie du jadis. (Quignard 2002, 79)

Il me semble essentiel de prendre en compte un argument qui n'apparait pas dans Dans ce jardin qu'on aimait ni dans les autres textes cités, mais qui est tout de même suggéré par la référence à la dépression saisonnière, c'est le rapport au réel : il faut avoir vécu pour avoir des choses à écrire. C'est la confrontation avec le réel, avec les aléas de la vie, qui pousse un être à écrire. Dans un entretien public avec la journaliste Raphaëlle Rérolle, Quignard a proposé plusieurs définitions du roman, dans l'une d'elles il suggère que c'est «l'expérience humaine qui fait le fond du roman » (Quignard 2011, 27). Pour approfondir et peut-être clarifier cette proposition, je me permets de citer Annie Ernaux qui, dans Mémoire de fille, développe l'idée d'un lien complexe entre écriture et expérience vécue, mal vécue le plus souvent :

C'est l'absence de sens de ce que l'on vit au moment où on le vit qui multiplie les possibilités d'écriture. [...] Explorer le gouffre entre l'effarante réalité de ce qui arrive, au moment où ça arrive et l'étrange irréalité que revêt, des années après, ce qui est arrivé. (Ernaux 2016, 151)

Ernaux exprime sans doute ici une des vérités de l'écriture de Quignard. Il pourrait y avoir une forme de proximité dans leurs démarches pourtant très différentes. Dans Les Larmes, Quignard matérialise son attachement, complexe, à la langue française, ainsi qu'à la littérature française et à leur histoire. Quignard donne une forme littéraire à ce lien si fort pour lui.

Pour terminer cette partie, je voudrais mentionner un critique littéraire, Jean Starobinski, qui s'est intéressé à la relation entre la vie d'un auteur et son œuvre. Les propos de Starobinski donnent un éclairage très intéressant à la démarche de Quignard, et à l'idée de l'écriture comme lutte contre la dépression, ou bien combat contre le sentiment d'absence de sens du vécu selon Ernaux. Pour Starobinski, l'écriture est en effet une expérience qui 
transforme la personne qui écrit, elle transforme le monde autour d'elle. Pour Starobinski, l'écriture "est l'expérience même » (Starobinski 2001, 40). Par le fait même d'écrire, l'auteur : «change le destin qu'il subissait comme être naturel » (Starobinski 2001, 324). En écrivant, le sujet devient acteur de sa vie, il n'est plus objet d'un destin qui s'impose à lui, mais il parvient à devenir un sujet qui peut assumer ce qui lui arrive. Starobinski ajoute que celui qui écrit « transforme la situation que lui assignait la société, [...] à la longue, il modifie la société elle-même. »(Starobinski 2001, 324) Ainsi, grâce à l'écriture, en affirmant sa résistance aux événements subis, le romancier produit des textes qui viennent concurrencer l'ordre du réel. L'écho qu'ils ont dans la société en vient à modifier le fonctionnement même de cette société.

On a là pratiquement un des thèmes principaux des Larmes. En donnant une forme écrite aux mots du peuple, Nithard transforme le monde, il change le destin des locuteurs du français et offre la possibilité à une littérature neuve d'émerger. L'écriture modifie la face du monde, Quignard a d'ailleurs publié un inédit intitulé « Le mot littérature est sans origine » où il affirme que «l'invention de l'écriture est plus importante que la découverte du feu » (Quignard 2015 a, 17). Nous allons retrouver la question de l'origine dans la dernière partie.

\section{Quignard et le réalisme}

Pascal Quignard n'est pas un auteur réaliste, son écriture ne s'inscrit pas dans une démarche d'imitation du réel, ni même de représentation de la réalité. Pourtant Les Larmes laisse une grande place à des événements historiques. Le roman s'appuie sur une série de faits historiques qui constituent une part non négligeable de l'œuvre. Il y a un décalage assumé entre la place de l'Histoire dans Les Larmes et la liberté d'un auteur qui invente et brode des aventures imaginaires dans une trame historique qui accumule des faits très précis, savants même. Je ne vais pas étudier cette question de manière approfondie, je souhaite seulement poser quelques repères qui permettront de mieux cerner la démarche de Quignard et de l'inscrire dans l'évolution générale de la littérature. Pour aller vite, pendant la période de la littérature réaliste, «le roman français a été un extraordinaire instrument d'exploration du réel » (Dubois 2000,9). Le roman était en mesure de dire la vérité du monde, de montrer ses rouages et les rapports de force qui existaient entre les différentes strates de la société. Le roman était capable d'imiter le monde, de reproduire la réalité grâce au travail de l'écrivain ; le roman pouvait même apparaitre comme plus vrai que le vrai. Cela fait que le roman était considéré comme un instrument de connaissance très précieux. Dubois distingue plusieurs étapes dans la conception du réalisme, il mentionne Stendhal qui, dans Le Rouge et le Noir, parle du roman comme d'un « miroir qui se promène sur une grande route. » (Stendhal 1830, 357 cité par Dubois 2000, 194). Plus tard, Maupassant soulignera les limites du réalisme, dans la préface de Pierre et Jean, il écrit :

Faire vrai consiste donc à donner l'illusion complète du vrai, suivant la logique ordinaire des faits, et non à les transcrire servilement dans le pêle-mêle de leur succession.

J'en conclu que les Réalistes de talent devraient s'appeler plutôt des Illusionnistes. (Maupassant 1889, 52 cité par Dubois 2000, 259)

Avec le temps, le roman devient, pour certains, un « espace de doute et le lieu d'une enquête pressente sur l'identité psychique et sociale des êtres » (Dubois 2000, 109). Puis de plus en plus de lecteurs considèrent que le roman est en grande partie « mû par un imaginaire 
dynamique, hanté par les désirs les plus variés et se construisant selon la symbolique latente d'un régime de pulsions » (Dubois 2000, 121).

Marcel Proust pense la littérature comme un espace de recherche où des sensations profondément enfouies dans les êtres peuvent revenir à la conscience par une écoute particulière. Proust va même jusqu'à écrire, dans Le Temps retrouvé, que : «La vraie vie, la vie enfin découverte et éclaircie, la seule vie par conséquent pleinement vécue, c'est la littérature »(Proust 1927, 202 cité par Dubois 2000, 276). Pour Proust, seul le processus littéraire est capable de procurer des révélations sur ce qui est ordinairement caché, inaccessible à la conscience. Selon lui, grâce à la littérature, la vie peut atteindre une forme de connaissance inégalée. Mais, dans le même temps, avec Proust, la notion de vérité perd de sa superbe, il n'y a plus de vérité établie, tout devient relatif, incertain, fuyant...

Par un tout autre biais, les recherches en linguistique ont mis en évidence l'arbitraire du signe. En France, un critique littéraire comme Roland Barthes a systématiquement mis en évidence la nature construite du langage, les lois auxquelles tout locuteur doit s'astreindre pour parler et échanger avec les autres. Barthes a été jusqu'à parler de tyrannie de la langue, et même de fascisme (Barthes 1977, 14).

Pascal Quignard, lecteur infatigable, connaît évidemment très bien toutes ces questions. Depuis la fin des années soixante, il est un acteur de la vie littéraire française : il est particulièrement conscient des enjeux qui se trament derrière l'écriture. Il a intégré, comme le rappelle Dubois, que «la fiction ne peut plus ignorer comme jadis ce qui fait sa définition essentielle et qui conjugue étroitement création de l'imaginaire et production de langage » (Dubois 2000, 332). Ainsi, on peut lire Les Larmes comme un travail sur le langage et comme une sorte de méditation sur la naissance de la langue française et de la littérature française.

\section{Esthétique de Quignard}

Je vais aborder le roman à partir des trois points énoncés dans l'introduction : sa forme, la fascination pour l'origine, le dédoublement des personnages principaux qui symbolisent deux types d'errance, la vie terrestre étant une forme d'exil pour Quignard. Dans Les Paradisiaques, ouvrage consacré au sentiment de perte du paradis que fut la vie intrautérine, Quignard décrit ce sentiment d'exil ressenti par beaucoup de ses personnages :

Enfants d'Ėve qui êtes exilés dans ce monde l'idée de paradis persiste dans ce simple souvenir d'avoir été nu dans ce lieu d'eau. [...] Le milieu in utero fut un Éden dans la mesure où il s'agissait d'un monde où le corps a été sans besoin. (Quignard 2005, 280)

\subsection{Une forme complexe}

Ce qui frappe dans ce court roman de 215 pages, c'est le découpage en chapitres très hétérogènes, dix chapitres divisés en de très nombreuses parties. Le texte ne joue pas sur l'ampleur romanesque mais, au contraire, sur un découpage précis et déroutant, car le texte passe d'un thème à un autre, d'une époque à une autre sans que l'on puisse saisir ce qui lie les différents éléments. Certes les jumeaux Nithard et Hartnid semblent les personnages principaux du roman, et l'invention de l'écriture du français et la naissance de la littérature française les thèmes principaux de l'œuvre. Mais Quignard donne l'impression de vouloir perdre son lecteur, et de le déstabiliser. 
Les Larmes mêle le discours historique convenu : le récit de la mort de Dagobert (Quignard 2016, 104), le couronnement de Charlemagne (Quignard 2016, 117), sa mort (Quignard 2016, 118), la mort de Nithard (Quignard 2016, 131), le mariage de Rollon et le gain de la Normandie :

En 911, à Saint-Clair, sur la rive de l'Epte, Charles le Simple donna à Rollon (Hrolfr) sa fille en mariage. Il lui cède toute la terre qui borde la mer, qui va de la Gaule belgique jusqu'à la frontière de la province de Bretagne. Cette longue et si riche et si belle laisse de mer et de terre, [...] fut appelée alors terre des Normands, ou Normandie. (Quignard 2016, 166-167)

On découvre aussi la présence plus inattendue d'un « je » qui livre des éléments de son histoire personnelle. Il y a, de manière troublante, une dimension proustienne dans l'évocation de l'étymologie des noms de lieu. Le narrateur exprime ses racines normandes :

Je suis né dans un pays où tous les noms se terminaient en bec ou beuf. Bec c'était le ruisseau. Beuf c'était la cabane. [...] Je vivais à Verneuil devant la ruine d'une église dédiée à saint Jean l'Évangéliste. (Quignard 2016, 52-53)

Le narrateur s'inscrit dans une sorte de filiation, il prétend écrire la langue que Nithard a inventée :

Même Nithard, qui était le plus lettré d'entre eux - en tout cas qui fut le premier d'entre eux puisqu'il écrivit pour la première fois la langue que maintenant j'écris, puisqu'il inventa cette langue en la notant un soir dans le camp dressé dans la neige sur la rive de l'Ill - (Quignard 2016, 82)

Dans une promenade, le narrateur semble changer de temps, la brume lui fait faire une sorte de voyage dans le temps, l'église retrouve ses origines vikings :

Tout à coup, tout d'un coup, je pénétrais la brume plus épaisse. Je marchais soudain plus lentement dans l'étrange ouate glacée. J'y voyais mal. Je marchais très lentement dans les taillis le long de l'eau. J'arrivais aux péniches de l'Yonne [...] Je passais devant la vieille église viking de Saint-Maurice où allait à la messe, enfant, Mallarmé. (Quignard 2016, 198-199)

On peut penser maintenant aux romans de Patrick Modiano, un personnage se promène dans un lieu et plusieurs temps se superposent; le présent de la promenade et un passé fantasmé qui surgit de l'imaginaire du personnage. Chez Quignard, l'érudition est constamment présente, l'imaginaire ramène à l'histoire de la littérature ou à des figures d'écrivains, cette même église viking a vu Mallarmé enfant.

Dans Les Larmes, le lecteur retrouve un personnage rencontré dans Les Paradisiaques (Quignard 2005) le frère Lucius. Son personnage est plus développé dans le roman, mais il lui arrive la même aventure, il disparaît pendant 300 ans en écoutant le chant d'un oiseau (Quignard 2016, 210-214). Le roman ouvre un espace où le rationnel s'estompe. Le personnage de Sar, chamane, prophétesse, illustre cette ouverture vers un monde où le fantastique a tous les droits. Nous sommes alors bien loin d'une présentation convenue de l'histoire officielle, d'autres forces opèrent dans le roman. On peut supposer que Quignard donne libre cours à ses fantasmes, il laisse son imaginaire s'exprimer. 


\subsection{La fascination pour l'origine et la naissance}

En apparence, les rédactions des «Serments de Strasbourg» et de la «Séquence de sainte Eulalie » sont présentées avec toute la rigueur de la science historique, Quignard revient en détail sur le contexte de production de textes fondamentaux qui ont changé le cours de l'histoire.

Nithard note dans son livre, à la date du 18 octobre 841, à 6h57, la merveilleuse étrangeté
qu'il découvre au-dessus des cimes des arbres du bois de Saint-Cloud. C'est une éclipse
de soleil. Sur quoi il termine le deuxième livre de son Histoire. [...] C'est alors que, le
vendredi 14 février 842, à la fin de la matinée, dans le froid, une étrange brume se lève
sur leurs lèvres. / On appelle cela le français. // Nithard, le premier, écrivit le français.
(Quignard 2016, 122-123)

Après les faits historiques que Quignard réécrit à sa façon, - était-il réellement 6h57 quand Nithard a écrit sa note? -, Quignard livre un commentaire personnel :

Rares les sociétés qui connaissent l'instant de bascule du symbolique: la date de naissance de leur langue, les circonstances, le lieu, temps qu'il faisait.

Le hasard d'une origine.

Il y a quelque chose de miraculeux de pouvoir observer le chiffrage. De pouvoir contempler le moment fou de transfert littéral. (Quignard 2016, 123)

Quignard souligne ce qui le fascine dans le fait de posséder une trace de la naissance de l'écriture de la langue, il y voit « quelque chose de miraculeux ». Il insiste et enchaîne des commentaires qui peuvent inclure un $« \mathrm{je} »$ :

Je vais être le plus précis possible tant cette naissance fortuite laisse stupéfait, délimite les terres, métamorphose le cours du temps. Le vendredi 14 février 842, à la fin de la matinée, en un seul mouvement on gravit sept paliers en un instant. (Quignard 2016, 124)

Ou bien, tirer des perspectives qui mettent en évidence l'importance de l'événement dans la pensée de l'auteur :

Ainsi, un jour d'hiver, un vendredi, le français et l'allemand se retrouvent-ils côte à côte à la fois dans une plaine d'Alsace et à l'intérieur d'une chronique qui, elle, est rédigée en latin, sous la plume d'oie de Nithard, le secrétaire palatial, sur une peau de veau soigneusement épilée et raclée. C'est la pierre de Rosette trilingue de l'Europe. (Quignard 2016, 125)

Les Serments de Strasbourg sont comparés à la pierre de Rosette, Quignard n'est pas exactement Champollion, d'autres avant lui ont traduit et commenté le manuscrit, mais Quignard donne toute sa valeur au texte. En mettant en avant Nithard, le rédacteur des Serments, il suggère qu'il a retrouvé le graveur de la pierre de Rosette. Il inscrit le texte dans une lignée familiale, dans une filiation qui l'inclut lui-même. En effet, ses propres grandsparents, philologues, professeurs à la Sorbonne, ont eux-mêmes contribué à la reconnaissance historique des Serments de Strasbourg.

Quignard procède sensiblement de la même manière avec la «Séquence de sainte Eulalie », il décrit les conditions objectives de la naissance du texte : «La tradition a intitulé 
ce premier poème écrit en français Séquence de sainte Eulalie. » (Quignard 2016, 147-148) Il explique aussi comment une copie a été retrouvée :

Trois ans plus tard, très précisément le mercredi 12 février 881, en préparation de la Procession et de la Fête annuelle de sainte Eulalie, la cantilène latine qui avait été dédiée à Sancta Eulalia fut traduite en français (in lingua romana) pour que tous les fidèles qui participaient au cortège, [...] puissent l'entonner sans qu'ils éprouvent de difficulté à saisir le sens de leur chant. [...] Il fallut attendre l'année 1837, dans la bibliothèque de Valenciennes, pour qu'un érudit remarque ces vingt-neuf vers français qui avaient été recopiés au début du mois de février 881, au terme du recueil, sur l'avers de la page de peau nue de cerf qui l'achevait. (Quignard 2016, 149)

Il raconte ensuite l'incendie de l'abbaye de Saint-Riquier :

À la fin du mois de février 881 l'abbaye de Saint-Riquier est mise à sac par les marins normands qui sont aussi d'impitoyables guerriers.

Plus de cent moines sur les trois cents qu'avait rassemblés Angilbert sont mis à mort. La bibliothèque est en partie incendiée mais toutes les peaux, étant épaisses, ne s'y consument pas. // Le premier livre où notre langue fut écrite est le premier livre brûlé de notre langue. (Quignard 2016, 153-154)

Cet épisode suggère que le premier livre a subi un autodafé, qu'il a donc expérimenté ce que d'autres livres vont subir après lui. La littérature, à peine née, est déjà menacée dans son existence matérielle. Des forces cherchent à la détruire. Il n'est peut-être pas inutile de rappeler que Quignard a souffert de la destruction de ses propres livres lors d'un salon du livre. Il a décrit cet événement déstabilisant dans Critique du jugement (Quignard 2015, 79).

Ensuite Quignard passe à un commentaire beaucoup plus personnel sur l'œuvre, il écrit par exemple : «Le français sort du latin comme un enfant du sexe de sa mère : comme un oiseau du cou de la sainte. » (Quignard 2016, 152). Les lecteurs de Quignard reconnaitront ici des obsessions de l'auteur, la relation entre le français et le latin, le rapport à la mère, à la sexualité, l'idée du texte littéraire comme porteur d'un sens caché que Quignard décrypte...

\subsection{Dédoublement de personagges}

Les livres d'histoire mentionnent l'existence d'un Hartnid, frère de Nithard, fils de Berthe et Angilbert. Dans Les Larmes, Quignard fait des frères des jumeaux et il oppose leur destin. On retrouve une opposition qui lui tient à cœur, on peut supposer qu'il respecte globalement la biographie de Nithard qui est plus documentée que celle de son frère, et qu'il invente une existence à Hartnid. Nithard a une vie monacale, il vit dans les livres, Hartnid au contraire a une vie pleine ; il voyage, rencontre des femmes, tombe amoureux :

Hartnid quant à lui faisait ce que son jumeau peinait à requérir, accomplissait ce qu'il s'épuisait à imaginer vainement, réalisait immédiatement tout ce que sa convoitise appelait de ses vœux.

L'un laissait à l'autre la part qui complétait son rêve.

L'un écrivait les deux pieds au chaud sur le couvercle de la boîte aux croisillons de fer qui recouvrait la braise. [...] L'autre naviguait, chevauchait, assouvissait ses envies, ses peurs, ses dégoûts, ses hontes, à l'autre bout du monde, de l'autre côté du monde. (Quignard 2016, 83) 
Quignard esquisse deux parcours de vie que sans doute il trouve fascinants. Dans de nombreux textes, Quignard présente les êtres humains comme des exilés, des êtres chassés du ventre de leur mère, le paradis du premier monde. On l'a noté dans Les Paradisiaques (Quignard 2005). Avec Nithard et Hartnid, il dépeint deux types d'errance : Hartnid est un aventurier, un voyageur, il se laisse porter par ses désirs et ses peurs, il n'a pas honte de vivre ses émotions. Nithard est avant tout un lecteur, un errant assis, sa vie est dans les livres, il consacre son existence à l'écriture et à la lecture. Il y a une part de sacrifice dans ce choix de vie, il est condamné à l'imagination, au rêve ; il ne connaît pas le concret des expériences de son frère. On voit souvent dans les textes de Quignard cette nécessité, chez les créateurs, de rentrer en eux-mêmes pour pouvoir créer. C'est le cas, par exemple, de Charles Chenogne dans Le Salon $d u$ Wurtemberg (Quignard 1986) ; il doit s'isoler pour pouvoir se souvenir et écrire, mais la mise à l'écriture le dépasse et il souffre de cet isolement pourtant voulu.

\section{Conclusion}

J'ai essayé de démêler quelques fils contenus dans le roman Les Larmes de Pascal Quignard. Je n'ai pas cherché à aplanir les difficultés de cette œuvre. Quignard fait du Quignard. Il s'inspire de la naissance de l'écriture de la langue et de la naissance de la littérature française pour créer une œuvre déroutante, hétérogène, où l'Histoire se mêle à l'imaginaire de l'auteur, inventeur d'une forme nouvelle.

J'ai évoqué Jean Starobinski qui suggère qu'écrire est une manière de devenir acteur de sa vie, d'échapper à un destin (Starobinski 2001). Je n'ai pas mentionné Paul Ricœur qui, dans Temps et récit (Ricœur 1983-1985), aborde la question complexe des liens entre Histoire, temps et fiction; comment le récit peut configurer et refigurer le temps. Ricœur forge la notion d'identité narrative (Ricœur 1985, 442) au terme de son étude pour indiquer qu'une identité se construit aussi par la fabrication de récits. Il serait intéressant d'analyser le roman de Quignard à partir des concepts de Ricœur, cela supposerait un examen méthodique de la pensée du philosophe. Mon approche était différente.

Je me suis efforcé de montrer que la démarche de Quignard est tournée vers le passé, l'origine, la naissance, que l'auteur est fasciné par les commencements et ce qui procède d'une genèse. Il est cependant possible d'interroger autrement le rapport de la littérature au réel, et de se tourner vers l'avenir pour le questionner. Dans Le Titanic fera naufrage (Bayard 2016), Pierre Bayard pose ouvertement la question de l'anticipation, un artiste est-il capable de décrire des événements qui se produiront plus tard ? Bayard analyse le roman de Morgan Robertson, Le Naufrage du Titan [Futility] (1898) qui décrit avec précision le naufrage du Titanic qui surviendra quatorze ans plus tard. Bayard commente aussi le film de Jean-Luc Godard, La Chinoise, tourné en 1967, mais qui annonce mai 68. Bayard ne prend pas position entre sceptiques et tenants de la précognition, mais il interroge les pouvoirs de l'imagination humaine.

\section{Bibliographie}

Barthes, Roland. 1977. Leçon. Paris : Seuil.

Bayard, Pierre. 2016. Le Titanic fera naufrage. Paris : Les Éditions de Minuit. 
Corneau, Alain. 1991. Tous les matins du monde, film cinématographique. DD Productions.

Dubois, Jacques. 2000. Les romanciers du réel. De Balzac à Simenon. Paris : Seuil.

Ernaux, Annie. 2016. Mémoire de fille. Paris : Gallimard.

Lapeyre-Desmaison, Chantal. 2001. Mémoires de l'origine. Un essai sur Pascal Quignard. Paris : Les Flohic Éditeurs.

Maupassant, Guy de. 1889. Pierre et Jean, préface « Le roman ». Paris : Folio classique.

Proust, Marcel. 1927. Le Temps retrouvé. Paris : Folio classique.

Quignard, Pascal. 1986. Le Salon du Wurtemberg, roman. Paris : Gallimard.

- 1991. Tous les matins du monde, roman. Paris : Gallimard (Folio 2533).

- 1993. Le Nom sur le bout de la langue. Paris : POL (Folio 2698).

- 1996. La Haine de la musique. Paris : Calmann-Lévy (Folio 3008).

- 2002. Sur le jadis (Dernier royaume III). Paris : Grasset (Folio 4137).

- 2005. Les Paradisiaques (Dernier royaume IV). Paris : Grasset.

- 2011. «Pascal Quignard. Entretien avec Raphaëlle Rérolle», dans Assises du roman 2011. Paris : Christian Bourgeois Éditeur.

- 2015. Critique du jugement. Paris : Galilée.

- 2015 a. «Le mot littérature est sans origine », dans Doumet, Christian et Ogawa, Midori (dir.), Pascal Quignard. La littérature à son Orient. Presses universitaires de Vincennes, coll. «L'imaginaire du texte ».

- 2016. Les Larmes, roman. Paris : Grasset.

- 2017. Dans ce jardin qu'on aimait. Paris : Grasset.

Ricœur, Paul. 1983-1985. Temps et récit. Paris : Seuil, coll. «Points. Essais ».

Starobinski, Jean. 1970 et 2001. La relation critique. Paris : Gallimard, édition revue et augmentée.

Stendhal. 1830. Le Rouge et le Noir. Paris : Folio classique. 